\section{Brustkrebs: Rezidivmuster über 24 Jahre}

\section{Rezidive eines Mammakarzinoms treten besonders häufig in den ersten Jahren nach Therapie auf. Zu Spätrezidiven gab es bislang kaum Daten.}

etzt legte die International Breast Cancer Study Group Daten aus ihren ersten 5 Studien über eine Beobachtungszeit von 24 Jahren nach Diagnose vor: Angaben zu 4.105 Patientinnen mit frühem Brustkrebs (Studienteilnahme zwischen 1978 und 1985). Die chirurgische Thera-

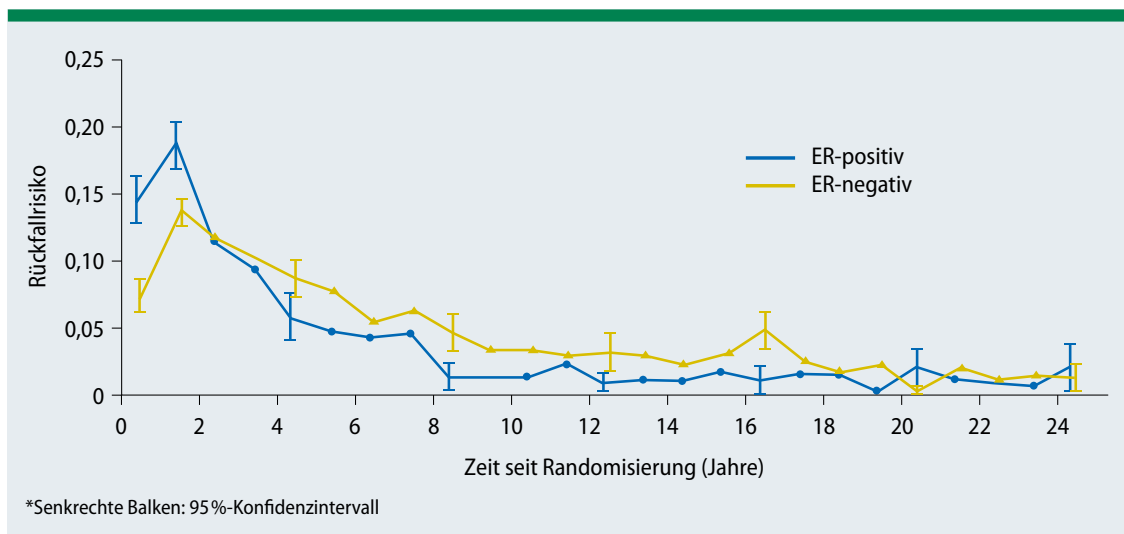

Abb. 1: Rückfallrisiko der Patientinnen aus den International Breast Cancer Study Group Trials I-V abhängig vom ER(„,estrogen receptor“)-Status ( $n=2.956)$

\section{Von Brustkrebspatientinnen berichtete Symptome bei ovarieller Suppression}

\author{
Im "Suppression of Ovarian Function Trial” ergab sich zumindest bei \\ Patientinnen mit vorangegangener Chemotherapie eine bessere Krankheits- \\ kontrolle durch die Suppression der ovariellen Funktion (OFS) zusätzlich zu \\ Tamoxifen. Nun liegen Ergebnisse zur Lebensqualität vor.
}

\footnotetext{
A usgewertet wurden Angaben zur Lebensqualität von 1.722 prämenopausalen Patientinnen mit Hormonrezeptorpositivem Brustkrebs, die randomisiert 5 Jahre Tamoxifen plus OFS ( $\mathrm{n}=861)$ oder Tamoxifen alleine $(\mathrm{n}=861)$ erhalten hatten. 474 Frauen im Kombinations- und 476 im Monotherapie-Arm hatten zuvor eine Chemotherapie erhalten. Die Fragebögen zur Lebensqualität („International Breast Cancer Study Group QoL Core Form" und ein studieneigener) waren $\mathrm{zu}$ Beginn, alle 6 Monate bis Monat 24 und
}

danach jährlich erhoben worden. Die mediane Beobachtungszeit betrug 5,6 Jahre.

Nach 6 und nach 24 Monaten ergab sich eine höhere Belastung durch Hitzewallungen bei den Patientinnen mit Tamoxifen plus OFS. Dies galt nach 6 Monaten auch für das sexuelle Interesse sowie Schlafstörungen und nach 60 Monaten für vaginale Trockenheit. Die Forscher bezeichneten die gynäkologischen Beschwerden aber als nur schwach klinisch relevant ausgeprägt. Dennoch brachen 19\% der Patientinnen unter Tam- krebs teilweise auch eine Oophorektomie. Lokoregional bestrahlt wurde nicht.

Das jährliche Rezidivrisiko in der gesamten Gruppe betrug in den ersten 5 Jahren 10,4\% - mit einem Gipfel zwischen den ersten beiden Jahren (12,5\%). In diesem Zeitraum war das jährliches Rezidivrisiko bei Östrogenrezeptor(ER)positiven Tumoren geringer als bei ERnegativen (9,9 vs. $11,5 \%$; $=0,01)$.

Das änderte sich im weiteren Verlauf. Zwischen dem 5.-10. Jahr betrug die jährlich Rezidivrate bei ER-positiven Tumoren $5,4 \%$, bei ER-negativen $3,3 \%$ und setzte sich in den weiteren 5-Jahres-Zeiträumen fort (Jahre 10-15: 2,9 bzw. 1,3\%; Jahre 15-20: 2,8 bzw. 1,2\%; Jahre 20-25: 1,3 bzw. 1,4\%). Damit bleibt das Rezidivrisiko bei ER-positiver Erkrankung dauerhaft erhöht.

Fazit: Patientinnen mit ER-positivem Brustkrebs weisen über lange Zeiträume ein erhöhtes Rezidivrisiko auf. Das bestätigt die Notwendigkeit einer langfristigen Nachsorge.

Friederike Klein

Colleoni M et al. Annual Hazard Rates of Recurrence for Breast Cancer During 24 Years of Follow-Up: Results From the International Breast Cancer Study Group Trials I to V. J Clin Oncol. 2016;34(9):927-35.

oxifen und $21 \%$ unter Tamoxifen plus OFS die Therapie frühzeitig ab. Bei vorheriger Chemotherapie schienen die symptomspezifischen Unterschiede zwischen den Behandlungsarmen geringer zu sein als ohne Chemotherapie. Die Gesamtwerte der Lebensqualität veränderten sich im Verlauf gegenüber dem Ausgangswert in beiden Studienarmen vergleichbar wenig.

Fazit: Insgesamt verursacht eine OFS zusätzlich zu Tamoxifen über die ersten 2 Jahre hinweg stärkere endokrine Symptome und eine beeinträchtigtere sexuelle Funktion als Tamoxifen alleine.

Friederike Klein

Ribi K et al. Adjuvant Tamoxifen Plus Ovarian Function Suppression Versus Tamoxifen Alone in Premenopausal Women With Early Breast Cancer: Patient-Reported Outcomes in the Suppression of Ovarian Function Trial. J Clin Oncol. 2016;34(14):1601-10. 